\title{
Le vote des agriculteurs aux élections professionnelles 1983-2007
}

The farmers' vote at the agricultural professional elections from 1983 to 2007

\section{Roger le Guen et Serge Cordellier}

\section{(2) OpenEdition}

1 Journals

\section{Édition électronique}

URL : http://journals.openedition.org/economierurale/811

DOI : 10.4000/economierurale.811

ISSN : 2105-2581

\section{Éditeur}

Société Française d'Économie Rurale (SFER)

\section{Édition imprimée}

Date de publication : 10 août 2009

Pagination : 14-31

ISSN : 0013-0559

\section{Référence électronique}

Roger le Guen et Serge Cordellier, « Le vote des agriculteurs aux élections professionnelles

1983-2007 », Économie rurale [En ligne], 312 | Juillet-août 2009, mis en ligne le 05 juillet 2011, consulté le 19 avril 2019. URL : http://journals.openedition.org/economierurale/811; DOI : 10.4000/ economierurale.811 


\section{Le vote des agriculteurs aux élections professionnelles 1983-2007}

Serge CORDELLIER • Chercheur indépendant

Roger LE GUEN • École supérieure d'Agriculture d'Angers

es votes des agriculteurs lors des trois Lélections aux Chambres d'agriculture organisées en 1989, 1995 et 2001 s'étaient progressivement et sensiblement déplacés des listes majoritaires FNSEA-CNJA vers celles de deux autres organisations syndicales, la Confédération paysanne (CP) et la Coordination rurale (CR), créées respectivement en 1987 et en 1994. Cette évolution avait souvent été interprétée comme la conséquence du décalage qu'exprimaient de plus en plus d'agriculteurs avec les orientations professionnelles dominantes, dans un contexte perçu en termes de ruptures tant des demandes sociétales que marchandes (Le Guen, 1997).

Les résultats du scrutin de 2007, qui ont vu les listes syndicales majoritaires progresser aux dépens de celle de la Confédération paysanne, ne peuvent donc être saisis dans la continuité d'une telle hypothèse. Deux types d'explications peuvent d'emblée être avancés : depuis 2001, soit les orientations des syndicats ont significativement évolué (celles des majoritaires et/ou des minoritaires), soit le rapport au contexte évoqué ci-dessus s'est transformé. Pour trancher entre ces deux hypothèses (leur association n'étant bien entendu pas à exclure), il faut, après avoir qualifié le déplacement des choix électoraux aussi précisément que possible, examiner à la fois l'évolution des programmes des différentes listes syndicales, celle des rapports des agriculteurs au contexte de leurs métiers, et les relations entre ces deux niveaux. Dans cet article est présenté un commentaire sociologique des résultats au collège des chefs d'exploitation (collège $n^{\circ} 1$ ) lors des élections aux Chambres d'agriculture départementales (1983-2007), qu'on comparera parfois à ceux des retraités « Anciens exploitants et assimilés » (collège $\left.n^{\circ} 4\right)$.

\section{Le cadre d'analyse}

En sciences politiques, l'analyse des comportements électoraux lors de consultations politiques est souvent fondée sur l'examen des rapports entre, d'une part, des offres de type idéologique représentées par des candidats et, d'autre part, des pratiques d'électeurs concernant un vote (le fait de participer ou non ; si oui, le fait d'exprimer un choix partisan). Nous considérerons de même que l'acte de voter résulte d'une activité de jugement porté à partir d'offres, dans des contextes spatio-temporels donnés. Ces offres reposent explicitement sur des programmes d'organisations (ici des syndicats) représentés par des listes de candidats qu'elles ont désignés. Mais cette activité de jugement des agriculteurs est aussi influencée par leur représentation des résultats obtenus lors de la mandature précédente à la Chambre départementale d'agriculture, et, au-delà, de ceux obtenus par les organisations syndicales. Résultats et organisations qu'ils connaissent plus ou moins précisément, dont ils se sentent plus ou moins acteurs, qu'ils peuvent identifier à des niveaux territoriaux différents (par exemple, localement, aux niveaux départemental, national). Les jugements étant en outre formulés dans un contexte de campagne électorale qui peut différer significativement de celui dans lequel avaient été obtenus ces résultats. Les pratiques électorales des agriculteurs lors des élections aux Chambres départementales d'agriculture doivent donc être envisagées, au-delà de simples choix idéologiques, comme des 
processus réflexifs et pratiques liés à des types d'engagement préexistants, à la fois dans leurs métiers et dans l'action professionnelle collective (ici dans des syndicats et en fonction de leur implication dans les domaines d'intervention des Chambres d'agriculture).

Nous considérons donc que l'activité de jugement d'un agriculteur lors du vote est liée à un processus d'engagement préexistant au plan syndical et, plus largement, professionnel. Le sociologue américain Becker (1960) considère l'engagement non pas comme la volonté calculatrice d'un sujet donné de satisfaire un intérêt, selon l'expression de Gardella et Monnet (2006), mais comme la recherche d'une cohérence dans le temps, concernant des buts et des choix de ce sujet qui sont étrangers à l'action dans laquelle il est impliqué à un moment donné. D'où la nécessité, pour Becker, d'analyser l'engagement comme un processus temporel placé dans un contexte historique donné. De son côté, le sociologue Thévenot (2006) propose le concept de « régime d'engagement » pour spécifier l'opération de jugement au moment de l'action inhérente à tout engagement, à travers laquelle les sujets évaluent, sélectionnent ce qui leur paraît pertinent pour agir. Il propose une démarche d'analyse de l'engagement assez similaire à la précédente, reposant sur l'étude du fonctionnement des interactions entre les personnes et leur environnement, dans une perspective dynamique.

Si le modèle d'analyse que nous avons développé à propos du vote professionnel de 2007 s'inscrit dans cette problématique, nous ne disposons pas des données nécessaires à une étude méthodique du lien entre les processus d'engagement des agriculteurs et leurs choix électoraux. Plus modestement, pour expliquer l'inflexion des choix professionnels des agriculteurs que représente le scrutin de 2007, nous avons mis en œuvre différents niveaux d'analyse qui demeurent empiriques et globaux concernant les mutations du corps électoral, les évolu- tions de la participation, les déplacements des scores syndicaux enregistrés ${ }^{1}$ et enfin les argumentaires syndicaux des élections de 2007 et leurs évolutions depuis 2001, resitués dans le contexte des marchés et de la politique agricoles.

\section{L'offre syndicale de 1946 à 2007}

Avant 1959, seules deux organisations syndicales nationales à vocation générale existaient : la Fédération nationale des syndicats d'exploitants agricoles (FNSEA), constituée en 1946, mais dont les racines puisaient dans une histoire remontant à la fin du xixe siècle; et le Centre national des jeunes agriculteurs, rebaptisé aujourd'hui JA - Jeunes Agriculteurs (CNJA), qui avait pris le statut syndical en 1956. En 1959, dans la mouvance de la gauche laïque, avait été créé le Modef (actuel Mouvement de défense des exploitants familiaux) et en 1969, la Fédération française de l'agriculture (FFA), sur des positions traditionnelles conservatrices. Jusqu'au début des années 1980, ce qu'on a pris I'habitude de nommer le " syndicalisme majoritaire " (FNSEA/[CN]JA) bénéficiait d'un monopole de représentation syndicale, car le Modef et la FFA n'étaient pas reconnus par l'État (ni au plan juridico-institutionnel, ni au plan politique). Lors de l'alternance politique de 1981, le gouvernement s'engagea dans la voie de la reconnaissance du pluralisme syndical, laquelle allait être lente, partielle et contrariée. Deux autres structures nationales nouvelles apparurent : la Confédération nationale des syndicats de travailleurs paysans (CNSTP), en 1981, et la Fédération nationale des syndicats paysans (FNSP), en 1982. Celles-ci allaient se réunir en 1987 dans la Confédération paysanne (CP). En 1994, enfin, fut créée la Coordination rurale (CR) qui avait absorbé la FFA mais n'en constituait pas un simple prolongement. On peut dire que les élections de janvier 2007 ont consacré ce pluralisme institutionnel.

\footnotetext{
1. Les résultats disponibles nous ont permis d'analyser les déplacements intervenus non seulement par rapport à 2001, mais aussi depuis le début des années 1980.
} 
Tableau 1. Évolution du nombre des inscrits pour les collèges " Exploitants " et « Anciens »

\begin{tabular}{l|c|c|c|c}
\hline \multicolumn{5}{|c}{ Chambres départementales (1983-2007) } \\
\hline & \multicolumn{2}{|c|}{ Exploitants et Assimilés } & \multicolumn{2}{c}{ Anciens Exploitants } \\
\hline Année & Effectif & Variation & Effectif & Variation \\
\hline $1983(1)$ & 1677253 & - & 879854 & - \\
\hline $1989(2)$ & 1360173 & $-18,90 \%$ & 1008369 & $+14,61 \%$ \\
\hline $1995(3)$ & 926170 & $-31,91 \%$ & 1125443 & $+11,61 \%$ \\
\hline $2001(4)$ & 691919 & $-25,29 \%$ & 1464266 & $+30,11 \%$ \\
\hline $2007(5)$ & 589144 & $-14,85 \%$ & 1349448 & $-7,84 \%$ \\
\hline$(5)-(1)$ & -1088109 & $-64,87 \%$ & +469594 & $+53,37 \%$ \\
\hline
\end{tabular}

\section{Contextes}

Au-delà des résultats proprement dits, l'étude du scrutin doit notamment être rapportée aux évolutions du corps électoral et à la participation par collège, d'autant que, d'un scrutin à l'autre, les variations de ces facteurs ont pu être considérables.

\section{Le corps électoral : des évolutions sociodémographiques profondes}

Préalablement à l'analyse des scores, il faut rappeler le renouvellement significatif des votants d'une élection professionnelle à l'autre, en particulier ceux du collège 1 des «Exploitants et assimilés », qui regroupe les chefs d'exploitations, qu'ils soient propriétaires, fermiers ou métayers, leurs conjoints, les aides familiaux et les associés d'exploitation $^{2}$ (tableau 1).

\section{Amenuisement et renouvellement}

Près d'un tiers des inscrits ont changé entre 2001 et 2007 : de $20 \%$ à $25 \%$ des électeurs ont quitté l'agriculture (la majorité d'entre eux changeant de collège, pour passer de celui des chefs d'exploitation à celui des retraités), tandis que $10 \%$ de nouveaux agriculteurs installés dans cet intervalle sont devenus de nouveaux inscrits. Il en résulte qu'au total, sur cette seule période, le corps électoral a baissé d'environ $15 \%$. Cette caractéristique est le résultat d'un taux élevé de départs compensé à moins de $50 \%$ par un faible taux de renouvellement.

2. En 1995, les pluriactifs dont l'activité agricole est seconde ont été intégrés au corps électoral.
Si l'on observe les évolutions pour les intervalles inter-scrutins précédents, on constate des diminutions encore plus importantes. Entre 1995 et 2001, le nombre des inscrits avait baissé d'un quart, avec une tendance plus accentuée dans la moitié ouest de la France, ainsi qu'en Alsace et que dans la partie méridionale de l'axe rhodanien. Le décrochage avait été sensiblement plus mesuré dans les régions Auvergne et Limousin et dans certains départements méditerranéens. L'évolution entre 1989 et 1995 avait été plus marquée encore, puisque le corps électoral avait diminué d'environ un tiers. Au total, en un quart de siècle (entre 1983 et 2007), le corps électoral du collège 1 a ainsi diminué de près des deux tiers et s'est profondément renouvelé.

\section{Évolutions des modèles professionnels}

Outre son caractère démographique, cette évolution traduit de profondes évolutions économiques et sociales. Les agriculteurs ayant cessé leur activité dans les décennies 1990 et 2000 correspondent en effet aux classes d'âge qui ont vécu la modernisation due à la politique des structures (effets des lois d'orientation et complémentaire de 1960 et 1962). Ils ont aussi connu la mise en œuvre puis l'extension de la Politique agricole commune (PAC) et le passage de la vulgarisation au développement agricoles (décrets de 1959 et 1966). Ces classes d'âge, dont les plus anciennes n'ont en majorité pas eu la possibilité de poursuivre des études (contrairement aux suivantes), ont été marquées par des débats professionnels très structurants, notamment, dans les régions 
RECHERCHES

Serge CORDELLIER, Roger LE GUEN

Tableau 2. Participation pour les collèges « exploitants ॥ [1] et « Anciens ॥ [4]

\begin{tabular}{c|c|c|c|c|c}
\hline \multicolumn{7}{c}{ Chambres départementales (1983-2007, en \%) } \\
\hline Années & 1983 & 1989 & 1995 & 2001 & 2007 \\
\hline Collège 1 & 68,35 & 58,52 & 57,80 & 61,22 & 66,40 \\
\hline Collège 4 & $38,18(1)$ & 33,63 & 37,07 & 39,00 & 47,90 \\
\hline
\end{tabular}

(1) Métropole seulement.

Source : ministère de l'Agriculture

devenues d'agriculture intensive : les débats de l'« âge d'or » de la modernisation, puis de la défense ou de la critique de celle-ci, dans le contexte d'un syndicalisme de masse exerçant un rôle de promotion sociale collective. Leur retrait a donc aussi correspondu à des changements de conceptions professionnelles et culturelles concernant le métier d'agriculteur ${ }^{3}$. Cette mutation correspond donc à de nettes inflexions dans le regard porté sur l'évolution de l'agriculture et son insertion dans l'économie. Il faut ajouter à ces éléments les changements intervenus dans les modèles de travail et de vie familiaux, notamment dans les processus de carrière (par exemple, le travail salarié avant installation) et les profils de couples (par exemple, la dissociation des métiers entre hommes et femmes), qui ont marqué les trente dernières années.

\section{Le taux de participation : prolongement de la reprise}

Le taux national de participation au scrutin de 2007 a progressé, passant de 61,2\% des inscrits à $66,4 \%$ dans le collège 1 . S'il avait toujours dépassé la moitié des inscrits, ce taux avait décliné de près de 10 points entre 1983 à 1989 après avoir, il est vrai, précédemment augmenté de plus de 11 points $(68,35 \%$ en 1983 , contre $56,63 \%$ en 1976 $1979)^{4}$. Cette augmentation avait suivi une réforme du mode de scrutin : la proportionnelle départementale de liste au plus fort reste avait remplacé un mode majoritaire de liste par circonscription électorale (avec renouvellement partiel tous les trois ans). Cette réforme, doublée des engagements

3. Voir notamment Rémy (1982) et Coulomb, Nallet (1980).

4. 57,0\% pour le renouvellement partiel de 1979 . gouvernementaux de reconnaître le pluralisme de représentation syndicale, avait créé les conditions d'une compétition plus ouverte et plus âpre. Le mode en vigueur en 2007 correspondait, pour le collège des « exploitants » et pour les deux collèges de salariés, à un scrutin majoritaire avec répartition proportionnelle suivant la règle de la plus forte moyenne (décret du 22 juillet 2000).

La progression du taux de participation n'a pas été spécifique au scrutin de 2007 : celle constatée lors des élections de la Mutualité sociale agricole (MSA) de 2005 a été plus forte encore, passant pour les « exploitants agricoles » (collège 1) de $44 \%$ en 1999 à $59 \%$ en $2005^{5}$. Cette progression ne peut non plus être globalement expliquée par les évolutions de l'offre syndicale, cette dernière étant resté assez stable au cours des 25 dernières années (312 listes en 1983, 291 en 1995, 294 en 2001 et 303 en 2007). On doit en revanche s'interroger sur l'impact de la technique de vote, laquelle a changé à l'occasion des élections aux Chambres de 2007, comme de celles à la MSA en 2005. L'instauration du vote par correspondance ouvert sur une période de quinze jours a sans doute simplifié et facilité la possibilité de voter. Lors des élections de 2007, on constate ainsi que le taux de votants du collège des retraités a lui aussi sensiblement progressé $(47,9 \%$ contre $39,0 \%$ ).

5. De même, $40 \%$ des salariés agricoles ont voté en 2005, contre $25 \%$ en 1999. Les taux de 2005 ont constitué des records dans l'histoire de la MSA, supérieurs de $10 \%$ à $20 \%$ aux taux de participation aux autres scrutins professionnels à la Sécurité sociale (source MSA, 2006). 
Enfin, on peut surtout expliquer la dynamique de mobilisation des agriculteurs exprimée par l'enjeu du scrutin lui-même. Car, contrairement à ce qu'affirment maints discours professionnels et savants qui soulignent depuis de nombreuses années la baisse du sentiment d'appartenance collective dans les organisations professionnelles agricoles, on peut poser l'hypothèse que ces élections ont traduit une volonté des agriculteurs de voir leurs intérêts collectivement représentés. Autrement dit, aux élections aux Chambres d'agriculture de 2007 comme à celles MSA de 2005 pour les « exploitants », l'augmentation de la participation des « chefs d'exploitation » traduirait, dans un contexte qui reste à préciser, une volonté accrue des agriculteurs de peser collectivement sur les orientations de leur profession.

\section{La nouvelle donne du scrutin de 2007}

Le scrutin de 2007 a permis aux listes FNSEA-JA non seulement d'enrayer l'érosion de leurs résultats, qui étaient passés d'environ 60,8\% en 1983 à 52,8 \% en 2001 (sans les listes dissidentes classées comme « apparentées » par le ministère de l'Agriculture), mais de progresser, à 55,2\%. La Confédération paysanne, en revanche, en a été la grande perdante, avec un score en recul de sept points, tandis que la Coordination rurale connaissait une nette reprise après sa stagnation de 2001. Cette reprise, qui l'a portée à près de $20 \%$, lui a permis de talonner la Confédération paysanne.

\section{Les résultats par organisation}

Matériel et méthode, quelques observations Nous avons tenté de mettre en perspective l'analyse des résultats des élections de 2007 avec ceux des scrutins précédents en nous appuyant sur les scores départementaux des listes syndicales et sur l'analyse des argumentations nationales des organisations. Certaines réunions prépa- ratoires ou postérieures à ces élections ont également été observées. Nos connaissances sociologiques et historiques des organisations syndicales départementales ont été mobilisées, car l'histoire et la trajectoire des organisations à ce niveau sont essentielles à la compréhension de leurs évolutions et de leurs modes de fonctionnement. Du fait du changement de mode de vote (par correspondance, les bulletins devant être adressés à la préfecture), on se trouve pour 2007 devant une absence totale d'information pour analyser sociologiquement les résultats du scrutin à l'échelle infra-départementale, niveau géographique où s'expriment des différences internes souvent plus fortes que celles observables entre départements. De ce fait, les comparaisons avec les scrutins antérieurs sont devenues impossibles pour ces échelons ${ }^{6}$.

En revanche, nous avons rationalisé la présentation des résultats départementaux et nationaux pour rendre leurs comparaisons plus objectives sur un quart de siècle (dans cette même section intitulée « La nouvelle donne », voir le point 2 «Une mise en perspective »). Le tableau 3 en est le résultat. Il représente un apport original à la connaissance du vote des agriculteurs aux Chambres consulaires.

\section{Succès pour les listes FNSEA/JA}

Les listes FNSEA et JA sont donc arrivées nettement en tête, progressant significativement de 2,4 points à 55,2 \% par rapport à 2001, année où elles avaient essuyé un très sensible revers par rapport à 1995

6. On regrettera par ailleurs, pour l'intérêt de la Recherche comme pour la connaissance de l'évolution de la société et de ses corps intermédiaires, les réductions, au ministère de l'Agriculture, des moyens humains du Bureau des Chambres d'agriculture et de leur Assemblée permanente, réduction qui, malgré le dévouement et la disponibilité de ses agents, freine le rythme de mise à disposition des données et en limite le volume. 
RECHERCHES

Serge CORDELLIER, Roger LE GUEN

Tableau 3. Résultats des listes du collège « Exploitants » 1983-2007 (en \%)

\begin{tabular}{l|c|c|c|c|c}
\hline Années & 1983 & 1989 & 1995 & 2001 & 2007 \\
\hline CNSTP [1] & 7,08 & - & - & - & - \\
\hline FNSP [2] & 5,80 & - & - & - & - \\
\hline Confédération paysanne [3] & - & 18,33 & 20,57 & 27,75 & 20,05 \\
\hline FFA [4] & 5,89 & 5,19 & - & - & - \\
\hline Coordination rurale [5] & - & - & 12,17 & 12,15 & 18,70 \\
\hline FNSEA-(CN)JA [6] & $60,72[8]$ & $62,89[9]$ & 56,44 & 52,80 & 55,19 \\
\hline Modef [7] & 9,84 & 7,86 & 5,05 & 3,62 & 2,92 \\
\hline Divers & 10,67 & $5,73[10]$ & 5,76 & 3,68 & 3,15 \\
\hline
\end{tabular}

[1] Confédération nationale des syndicats de travailleurs paysans, qui sera cofondatrice de la Confédération paysanne en 1987 (taux incluant les scores des listes d'alliances intersyndicales) ; [2] Fédération nationale des syndicats paysans, qui sera cofondatrice de la Confédération paysanne en 1987 (taux incluant les scores des listes d'alliances intersyndicales) ; [3] En tenant compte des listes d'alliance avec d'autres organisations, notamment le Modef ; [4] Fédération française de l'agriculture, qui sera absorbée par la Coordination rurale en 1994 ; [5] Créée en 1994 et ayant absorbé la FFA ; [6] Pour tous les scrutins, une seule liste revendiquée par l'union FNSEA-(CN)JA a été prise en compte. Ces taux ne tiennent donc pas compte des listes classées, depuis 1995, comme « apparentées » par le Ministère (3,35 \% en 1995, 1,39 \% en 2001, $1,44 \%$ en 2007) ; [7] En tenant compte des listes d'union avec la Confédération paysanne ; [8] 61,1 \% selon les résultats officiellement proclamés par le ministère de l'Agriculture ; [9] 64,3\% selon les résultats officiellement proclamés par le ministère de l'Agriculture qui comptabilisent une seconde liste pour certain départements ; [10] Incluant les scores de deux listes départementales (Morbihan, Gard) totalisant 1,34 \% du total des suffrages exprimés au plan national et classées « Non classées » par le Ministère car ayant fait l'objet de revendications concurrentes.

Sources : résultats publiés par le ministère de l'Agriculture et calculs de l'auteur

(- 3,4 points $)^{7}$. En 2007, les listes FNSEAJA ont gagné des voix dans 62 départements et ont progressé d'au moins $15 \%$ dans 24 d'entre eux (principalement dans le Centre et la Bourgogne, une partie du NordEst et du Sud-Est). Elles ont en revanche perdu des voix dans 32 autres départements, situés surtout dans la bordure septentrionale du Bassin parisien, le Centre-Est et dans le Sud. Trois fois sur quatre, la baisse a cependant été inférieure à $15 \%$. Dans la moitié des départements en baisse, la Coordination rurale présentait une liste pour la première fois.

La conquête de la Chambre d'agriculture de Loire-Atlantique, qui était jusqu'alors détenue par la Confédération paysanne a marqué une victoire symbolique

7. En 2001, le score des listes FNSEA-CNJA avait baissé dans 69 départements sur 94, la diminution dépassant $30 \%$ des suffrages exprimés dans 11 d'entre eux. L'échec de 2001 faisant lui-même suite à un très sévère repli de 6,4 points entre 1989 et 1995. importante pour la FNSEA. À partir de 1976, cette Chambre avait en effet été dirigée par un courant (majoritaire) de la FDSEA qui, après son exclusion par la FNSEA en 1978, allait cofonder la Fédération nationale des syndicats paysans (FNSP). Puis, avec les travailleurs-paysans du département, elle avait participé à la création de la Confédération paysanne. Le département avait, depuis la fin des années 1960, représenté une sorte de place forte de la « gauche paysanne » (Bourrigaud, 2001), avec des leaders nationaux d'envergure tels que Bernard Lambert (1931-1984) et Bernard Thareau (1936-1995).

\section{Un échec pour la Confédération paysanne}

La Confédération paysanne (CP), qui a conservé de peu le deuxième rang pour la représentativité, a très nettement reculé : elle a perdu un tiers de voix par rapport à 2001 (72 708 contre 109 041) et son score (en incluant les listes d'unions intersyndicales) a baissé d'un quart, passant de $27,7 \%$ à $20,1 \%$ des suffrages exprimés. Elle a 
ainsi annulé ses très importants gains de $2001^{8}$ et est revenue à son niveau de 1995. La baisse a été générale et a semblé indépendante de l'ancienneté et de l'importance de l'implantation, ainsi que des pratiques syndicales locales. Les listes de la CP ont reculé dans 77 départements où elles étaient présentes en 2001, et de plus de $15 \%$ des voix dans 68 d'entre eux. Elles n'ont progressé que dans quatre départements situés au sud de la France, ainsi qu'à La Réunion où a été enregistré un score exceptionnel $(72,8 \%)$ avec gain de la présidence de la Chambre. La CP a en revanche perdu celle de la Guyane. Elle a aussi perdu, comme on l'a vu, celle de Loire-Atlantique.

Incontestablement, le recul de la CP s'est principalement réalisé au profit de la Coordination rurale (CR). Il semble aussi qu'après les retombées médiatiques considérables de l' « effet Bové » qui avaient, en 2001, favorisé le succès de la Confédération par des discours et mobilisations concernant l'alimentation et le commerce international des produits agricoles, une partie des électeurs ont manifesté leur désaccord avec certaines postures publiques de l'ancien leader syndical (José Bové) auquel, qu'elle l'ait voulu ou non, l'image extérieure de la CP était restée fortement attachée ${ }^{9}$. Par ailleurs, la désaffection électorale est sans

\footnotetext{
8. Elle avait progressé dans 56 départements où elle était déjà présente en 1995 (de plus de $30 \%$ dans 41 ), obtenant pour la première fois la représentativité dans nombre d'entre eux. Ces résultats n'avaient que rarement fait l'objet d'une analyse électorale exigeante et peu d'observateurs avaient souligné, par exemple, que de nombreuses listes départementales de l'Ouest avaient vu leurs résultats baisser.

9. Ces postures ont notamment concerné son engagement dans la campagne pour le «non » lors du référendum portant sur le traité constitutionnel européen en 2005 (option assumée par l'organisation) et, bien sûr, sa candidature et sa pré-campagne pour l'élection présidentielle de 2007 (qui, elle, n'engageait pas la (P), deux événements à l'occasion desquels il est apparu politiquement "positionné » à l'extrême gauche.
}

doute aussi venue du sentiment que cette organisation, très présente dans les médias pour ses engagements altermondialistes, en avait peut-être oublié les tâches plus « traditionnelles » de défense syndicale en termes d'action professionnelle (dans la section ciaprès intitulée « Amorce d'une interprétation sociologique », voir « Identité professionnelle »). Une motion présentée par les régions Bretagne, Basse-Normandie et Poitou-Charentes, adoptée par le congrès de mai 2007 (Le Mans), affirmera ainsi que c'est « en revenant sur [le] terrain des préoccupations des paysans que nous pourrons être convaincants et efficaces dans une campagne d'adhésion, indispensable à la survie de notre organisation syndicale ».

\section{Coordination rurale :}

les fruits d'une implantation élargie

La Coordination rurale (CR) s'est nettement renforcée, passant de $12,1 \%$ à $18,7 \%$ des voix. Elle a progressé dans 31 départements sur les 53 où elle était déjà présente en 2001 (de plus de $15 \%$ des voix dans 24 d'entre eux). Mais elle s'est affaiblie dans 22 autres, situés notamment dans les zones de grandes cultures, là où elle avait progressé en 2001. Elle a conservé les deux Chambres d'agriculture qu'elle présidait, depuis 1995 pour le Calvados et depuis 2001 pour le Lot-et-Garonne. Le score de la Coordination rurale s'explique essentiellement par le fait que, cette fois, elle a été en mesure de présenter 80 listes départementales homogènes, contre 53 en 2001, soit une augmentation de plus de moitié. Cela a mécaniquement engendré une progression de son score d'environ 6 points de pourcentage. Le résultat de la CR correspond donc à un réel progrès global, mais non pas à un progrès en score moyen par liste.

La géographie de l'implantation de cette organisation est très lisible. Créée sous forme syndicale en 1994, mais ayant bénéficié de l'acquis de l'implantation de la FFA, ses zones de force apparaissaient à l'origine principalement concentrées dans les 
zones de grandes cultures du Bassin parisien (Eure-et-Loir, Indre-et-Loire, Loiret, Seineet-Marne, etc.) et en Poitou-Charentes - où son lancement (au début de 1992, avant qu'elle ne prenne le statut syndical) avait bénéficié de l'alliance avec le Modef et la Confédération paysanne. Elle possédait par ailleurs une implantation en Pays de la Loire et, pour partie, en Aquitaine, Midi-Pyrénées et Bourgogne. En revanche, elle était absente ou très faiblement présente dans le Grand Massif central, en Bretagne (sauf dans le Morbihan, héritage de la FFA) et sur les frontières est de la France, de Lille à Toulon en passant par les Deux Savoies. La CR n'avait pas été capable d'étendre significativement son implantation en 2001 mais, en revanche, elle a été en mesure de se présenter en 2007 sur l'essentiel du territoire métropolitain, ce qui a été pour elle un incontestable succès politique.

\section{Le faible étiage du Modef}

Le Modef, pour sa part, est resté à son faible étiage $(2,9 \%$ contre $3,6 \%$ en 2001 , en comptant les listes d'union), mais avec un nombre de listes nettement moindre : 21 listes homogènes contre 33 en 2001 et 40 en 1995. Il a néanmoins progressé dans sept départements et bien résisté dans son fief des Landes (31,6 \%). Il n'a pratiquement présenté de listes que dans la moitié sud de la France et dans les DOM. La carte de cette organisation, en fort déclin depuis le début des années 1980, dessine depuis sa création un territoire d'affinité fidèle, pour l'essentiel situé au sud de la Loire sur des terres historiquement laïques et républicaines (Charentes, Limousin, Sud-Ouest, Sud-Est...). En 1983, où il avait enregistré un score national de $8,8 \%$ en moyenne nationale (faisant suite à 18,8 \% en 1976-1979, soit une chute brutale de moitié), ses points forts apparaissaient ainsi être les Landes (48,5\%), le Tarn-et-Garonne (28,5\%), la Charente, l'Aude, le Vaucluse. Le Modef bénéficiait par ailleurs d'implantations significatives en Charente-Maritime, Corrèze,
Pyrénées-Orientales, dans le Var, l'Ardèche, la Drôme et les Hautes-Alpes, départements où il obtenait entre $20 \%$ et $25 \%$ des suffrages exprimés. L'électorat du Modef a semblé s'être progressivement «évaporé », de même que sa base sociale historique de petits exploitants, mais la mémoire de son influence reste visible au travers des scores très significatifs que ses candidats recueillent dans le collège des « anciens exploitants » dans certains départements d'implantation historique. C'est ainsi qu'en 2007, une vingtaine de ses listes ont dépassé $20 \%$ et une dizaine $30 \%$. Sans compter celles présentées, dans d'autres départements, par une organisation proche, l'Association nationale des retraités agricole de France (ANRAF), dont les résultats ne peuvent cependant pas être globalement assimilés à un vote Modef.

\section{Une mise en perspective}

Il convient d'étudier, à l'échelle interdépartementale, les raisons des transferts de voix des listes de la Confédération paysanne vers la Coordination rurale et, de manière beaucoup plus limitée, vers le couple FNSEA-JA ; ainsi que ceux, moindres également, des listes de la Coordination rurale vers les listes FNSEA-JA.

\section{Votes d'adhésion et votes d'expression}

Il est manifeste, à l'observation des différentes configurations départementales, que la Coordination, là où elle n'était pas présente en 2001, a nettement plus mordu sur l'électorat de la Confédération paysanne que sur celui de la FNSEA. Cela a été le cas, par exemple, dans le Finistère, où la $\mathrm{CP}$ a perdu 11,2 points, tandis que la CR recueillait $16,9 \%$ des suffrages - la FNSEA a perdu pour sa part 2,8 points tandis que le Modef ne se représentait pas $(2,9 \%$ en 2001). Cela a aussi été le cas, bien plus spectaculaire, dans les départements des deux leaders altermondialistes les plus connus de la Confédération, l'Aveyron de José Bové (le score de la CP y a été divisé 
par deux, passant de 44,0 \% à 22,8 \%) et la Manche de François Dufour (de 46,4 \% à $23,9 \%$ ) tandis que la CR recueillait respectivement $21,4 \%$ et $13,4 \%$. La FNSEA est restée stable en Aveyron mais a progressé de près de 10 points dans la Manche ${ }^{10}$.

On peut penser que la Confédération paysanne, organisation fondée en 1987, aura bénéficié dans les années 1990 d'une « rente d'opposition » à la FNSEA, comme cela avait été auparavant le cas pour le Modef dans les années 1970 (avec, à cette époque, des scores moyens atteignant fréquemment $30 \%$ là où il se présentait). Opposée à la fois à la «politique des structures » et à la construction européenne, cette organisation avait joué à cette époque une «fonction tribunicienne » pour la petite paysannerie, pour reprendre l'interprétation féconde du politologue Georges Lavau à propos du mouvement communiste (Lavau, 1981). Désormais, avec la Coordination rurale, l'offre syndicale est élargie et le mécontentement et les aspirations au changement peuvent s'exprimer aussi en votant pour une organisation qui n'est soupçonnée ni d'être marquée à gauche ni d'entretenir des sympathies pour l'écologie politique, tout en étant oppositionnelle à la FNSEA. La Coordination, en prônant à la fois le protectionnisme commercial et la liberté dans la politique des structures, le tout sur fond de ruralisme politique, est à son tour en mesure de capter une partie du vote d'opposition à l'État et/ou au syndicalisme majoritaire, mais sa performance ne se limite pas à cela.

L'analyse des résultats fait par ailleurs apparaître qu'au-delà du choix d'une préférence syndicale, il existe aussi un vote antiFNSEA qui s'est porté, selon les configurations locales, sur quatre catégories de listes : Confédération, Coordination, listes « dissi-

10. Il faut noter que si la Coordination était absente de ces deux départements en 2001, elle était présente dans la Manche en 1995 (8,0\%), ainsi qu'une liste «dissidente » de la FDSEA (« Unité et forces paysannes »), qui avait atteint $16,6 \%$. dentes » de la FNSEA et des JA ou encore listes classées « diverses ». Les exemples abondent qui montrent que, d'un scrutin à l'autre, une partie de ces voix peut se déplacer d'une catégorie à l'autre.

Les scores syndicaux sur un quart de siècle En observant l'évolution du poids électoral des organisations syndicales sur un quart de siècle (voir le tableau 3), on constate, de 1989 à 2001, une érosion lente mais régulière des scores FNSEA-CNJA au profit de la Confédération paysanne et de la Coordination rurale, légèrement compensée en 2007 par un rebond du syndicalisme majoritaire.

Avant 1983 le scrutin reposait, comme on l'a vu, sur un renouvellement par moitié des élus tous les trois ans. D'autre part, les syndicats minoritaires n'avaient aucune représentativité nationale reconnue. Le scrutin de 1983 a été très particulier car il devait servir à étalonner la représentativité de toutes les organisations. Ce scrutin a été très disputé et très politisé, le gouvernement de gauche comme la FNSEA entendant en faire un test. Les résultats officiels avaient été obtenus après polémiques et reclassement de plusieurs listes. Le taux indiqué au tableau 3 pour 1983 et 1989 résulte d'une reconstruction méticuleuse et inédite effectuée par nos soins, département par département, liste par liste. Pour ce qui concerne l'union FNSEA-CNJA (comme pour les autres organisations), une seule liste a été retenue par département, la plus légitime en chaque cas, qu'elle soit favorable ou non à la direction de la FNSEA. Le score national ainsi calculé $(60,7 \%)$ pour cette dernière en 1983 est très proche, bien que de composition différente, de celui finalement proclamé par le ministère de l'Agriculture $(61,1 \%)$. La politisation très offensive de ce scrutin menée par François Guillaume à la tête de la FNSEA (19791986), avait abouti à des divisions internes à la centrale, affaiblissant fortement son score potentiel. Les listes revendiquant le label UFP (Unité et Force paysannes), dont 
elle avait fait son drapeau en alliance avec le CNJA et la Confédération nationale de la mutualité, de la coopération et du crédit agricoles (CNMCCA), n'avaient enregistré que $54,40 \%$ des suffrages exprimés (des listes se revendiquant de ce label avaient été présentées dans 79 départements métropolitains sur 90). En 1989, sous la présidence de Raymond Lacombe (1986-1992), la situation était plus apaisée et la FNSEA avait regagné deux points, avant de « décrocher » sérieusement en 1995, puis en 2001.

\section{Amorce d'une interprétation sociologique des résultats}

Pour expliquer l'inflexion des votes intervenue en 2007, nous avons identifié trois types de facteurs sociologiques, parfois mêlés :

- les argumentations développées par les organisations syndicales à propos des perspectives de développement économique et social du secteur agricole et les enjeux environnementaux qui s'y rattachent (valeurs, programmes, projets) ;

- les liens des organisations syndicales avec la mise en œuvre de la politique publique et avec l'ensemble de l'organisation professionnelle de l'agriculture tels qu'ils apparaissent dans leurs documents publics, mais aussi tels que nous avons pu les observer à l'occasion de certaines réunions électorales ;

- la concurrence et la compétition entre les organisations. Celles-ci concernent - en particulier pour la Confédération paysanne, la Coordination rurale et le Modef - d'une part, la capacité à capter et à répondre à l'expression des agriculteurs vis-à-vis des évolutions de la politique européenne et nationale et des positions du syndicalisme majoritaire et, d'autre part, leur réponse aux attentes de reconstruction d'un sens du métier, dans un contexte que beaucoup d'entre eux conçoivent en termes de rupture.

\section{Un net changement de contexte}

En 2001, les préoccupations des agriculteurs étaient fortement marquées par la répétition de crises sanitaires intervenues dans différents secteurs d'élevage en France comme en Europe, perçues comme autant de scandales ${ }^{11}$. Malgré les menaces de peste aviaire de 2006, ces crises n'étaient plus au centre de leurs préoccupations au moment des élections de 2007. Ce sont plutôt les conséquences de la réforme de la PAC qui mécontentaient beaucoup d'entre eux, pour des motifs parfois très différents ${ }^{12}$. D'autant que la réforme était annoncée comme étant une étape vers une réduction sensible des moyens de la PAC à l'horizon 2013. Enfin, la question des productions agricoles non alimentaires suscitait des espoirs chez les uns, des critiques chez d'autres. Bref, tout se passait comme si des préoccupations de type économique du métier avaient pris le pas sur celles de la période précédente, de type avant tout sociétal.

Au titre des contextes, un autre facteur, la bonne conjoncture économique de la plupart des grands marchés agricoles en 2006, notamment ceux des céréales et de la viande bovine, a pu contrebalancer le mécontentement des agriculteurs consécutif aux réformes de la PAC. Enfin, même si cet argument a été trop souvent et trop commodément évoqué par certains médias,

11. Ce furent les crises de l'encéphalopathie spongiforme bovine (ESB), dite « maladie de la vache folle », en 1996 et en 2000 ; l'affaire « du poulet à la dioxine » en 1999 ; la pandémie de fièvre aphteuse en Grande-Bretagne ayant touché l'Ouest de la France en 2001.

12. En 2005, selon une enquête sociologique nationale à laquelle nous avons participé, seuls $3 \%$ des agriculteurs considéraient la nouvelle PAC comme une bonne solution, 27,5\% «dans le cours des choses», plus de $29 \%$ comme négative pour l'avenir du métier, et $21 \%$ voulaient revenir à une politique protectionniste (In « Les aspirations des agriculteurs et leurs rapports à la profession », Rapport de synthèse d'une enquête nationale menée auprès de 505 agriculteurs », FESIA-FNSEA, juillet 2006, 222 p. + annexes). 
le contexte politique et idéologique des différentes élections politiques ne peut être totalement évacué.

\section{Une concurrence syndicale plus paisible}

Comprendre comment, depuis 2001, les agriculteurs ont interprété l'offre syndicale et ses évolutions suppose de caractériser la concurrence en son sein. Les relations entre listes sont apparues plus paisibles en 2007 qu'en 1995 et 2001 . Le discours public de la Coordination rurale a semblé moins protestataire qu'en 1995 lorsqu'elle dénonçait la « trahison » de la FNSEA à propos de la réforme de la PAC de 1992. Elle arguait alors que la centrale majoritaire bradait les intérêts des producteurs de grandes cultures et menaçait la vie rurale de régions entières. De leur côté, en 2001, les syndicats FNSEA et JA apparaissaient « aux abois » face aux crises alimentaires à répétition et aux débats sur les négociations à l'Organisation mondiale du commerce (OMC). Quant à elle, la Confédération paysanne, toute à son offensive syndicale et altermondialiste, était alors relayée par les grands médias autour de trois thématiques : l'insécurité sanitaire provoquée par l'industrialisation alimentaire, la «malbouffe » et les organismes génétiquement modifiés (OGM) présentés comme ses corollaires, et enfin la libéralisation des échanges agricoles. La CP avait su traduire positivement une partie des votes d'opposition au couple FNSEA-JA, en soulignant le déphasage entre la PAC, la politique de cogestion agricole et la montée des aspirations sociétales à une consommation alimentaire plus saine et à un commerce plus équitable.

\section{Des slogans devenus porteurs}

En 2007, l'écho de la plupart de ces thématiques semblait atténué. Et certains slogans de campagne affichaient cette fois de fortes ressemblances. En effet, en comparant les programmes et les rapports de congrès des dernières années, on a généralement retrouvé de mêmes slogans dans les discours de toutes les organisations, reprenant les notions de « souveraineté alimentaire », de « rémunération du travail », d' " agriculture paysanne », sans compter toute une série d'arguments critiques contre la mondialisation, l'OMC, le libéralisme et le libreéchange, etc. (Cordellier, 2006) ${ }^{13}$ La Confédération paysanne, qui avait historiquement construit sa stratégie autour de ces axes, a ainsi pu se croire imitée ou "récupérée ». Mais ce phénomène n'a-t-il pas d'abord traduit la promotion politique de ses idées et le fait d'avoir imposé des thèmes et des objectifs devenus légitimes pour tous ? Quitte à ce que d'autres organisations se contentent de reprendre son discours sans envisager la mise en œuvre qui en découlerait...

De leur côté, la FNSEA et JA se sont efforcés de présenter un projet fondé sur une double rhétorique ${ }^{14}$ :

- une thématique répondant aux critiques des autres syndicats : le prix et la qualité des produits, la résistance au libéralisme, la restauration de la préférence communautaire, la diversité des agriculteurs, la solidarité, l'installation, les « énergies vertes », l'animation des territoires ruraux ; - une vision positive de l'avenir du métier : répondre à la segmentation alimentaire, réguler les marchés, développer de nouveaux marchés non alimentaires, améliorer les conditions de compétitivité des entreprises.

En revanche, FNSEA et JA se sont bien gardés de s'exprimer sur les enjeux environnementaux de l'exercice du métier, ainsi que sur la répartition des aides publiques aux exploitations, deux dimensions

13. $C f$. Serge Cordellier, «Élections aux chambres d'agriculture : des programmes différents, mais des formules communes », TransRural Initiatives, n 324, 19 décembre 2006.

14. FNSEA, JA, Chambres d'agriculture 2007. Notre métier a un prix. Pour une agriculture audacieuse, attractive et solidaire, septembre 2006. 
pourtant placées de plus en plus au centre des discussions politiques sur l'avenir de la $\mathrm{PAC}$, chères à la Confédération paysanne et régulièrement soulignées par les médias. Ces deux dimensions étaient en effet susceptibles de diviser leurs propres forces.

\section{Les argumentations sur le développement économique}

Entre 1995 et 2001, la Confédération paysanne avait été perçue comme formulant en quelque sorte mieux que les autres organisations le sens des crises sanitaires de l'agriculture et de l'alimentation, tant aux yeux de ses propres adhérents ou électeurs que des médias. Cette différence avait d'ailleurs été soulignée par de nombreux responsables et militants de la FNSEA et de JA dans des réunions syndicales nationales et départementales. Sur fond de dénonciation du processus d'industrialisation de l'agriculture et de l'alimentation, qui était accusé de constituer de graves menaces pour la santé des consommateurs et pour l'avenir des éleveurs, la $\mathrm{CP}$ proposait une orientation alternative, qualifiée d' " agriculture paysanne », projet qu'elle portait en théorie et en pratique depuis la fin des années 80 et qu'elle avait inscrit, dans les années 90, dans une stratégie de « développement durable ».

\section{OGM, agrocarburants...}

Avant le scrutin de 2007, notamment dans les premières années 2000, la Confédération était intervenue sur un mode nettement plus défensif, voire «négatif », sur la politique publique européenne (la nouvelle PAC était rejetée en tant que "système néfaste ») et sur les risques suscités selon elle par de nouvelles perspectives d'innovations technologiques (la CP s'opposant à la fois aux OGM et au développement des agrocarburants).

La direction élue en 2005, lors de l'Assemblée générale de 2006, avait certes affirmé la nécessité de mener de front contestations et propositions $^{15}$, mais ce souci d'équilibre avait été peu perçu hors du cœur de l'organisation et beaucoup d'électeurs ont vu la CP comme un syndicat du refus du changement, d'autant que la multiplication des procès à l'encontre de ses militants ou sympathisants, notamment pour les «fauchages d'OGM » était régulièrement venue alimenter les faits divers médiatiques.

Dans les débats politiques et professionnels portant sur l'avenir de l'agriculture, les craintes liées au démantèlement de la PAC, comme les espoirs de développement des agrocarburants, ont été portés principalement par la FNSEA et ses associations spécialisées. De ce fait, elles ont pu apparaître, d'un côté comme rempart protectionniste $^{16}$ et, de l'autre, comme les porteuses d'un grand projet de débouchés prometteurs pour les producteurs de grandes cultures, une sorte de relance bénéfique pour toute l'agriculture. Deux modèles s'opposaient, le premier, pouvant être qualifié de «fordiste », porté par la FNSEA (la production de blé et de colza, maillon d'une chaîne de « carburant vert »

15 « Faut-il choisir entre le grand soir et les petits matins? Il ne doit pas y avoir de contradiction [...], mais il y a parfois un décalage entre le discours et l'aspect concret des choses vécu par les paysans. [...] Nous devons rechercher le contact et l'interpellation des paysans [...]. » (Rapport moral. Projet de contribution au débat stratégique, Assemblée générale du 19 avril 2006, cité par S. Cordellier, «Rééquilibrages et débats internes à la Confédération paysanne », TransRural Initiatives, $\mathrm{n}^{\circ} 310$, 23 mai 2006).

16. La condamnation, en décembre 2006 , de la FNSEA par la justice européenne à $12 \mathrm{M} €$ d'amende pour entente illicite, en raison d'un accord passé sur les prix de la viande bovine lors de la crise de l'ESB en 2001, avait fait apparaître l'organisation majoritaire comme défendant à la fois une « régulation interprofessionnelle » des crises de marché et comme « victime de l'idéologie libérale européenne ». 
appelée à une croissance très importante $)^{17}$; l'autre, que nous qualifierons de « domestique », porté par la Coordination rurale (la production d'un carburant à la ferme, voir local, échappant à l'emprise tant de l'État que des industries).

La Coordination rurale avait en effet choisi de ne pas chercher à tirer profit de la perspective de développement des agrocarburants. Elle dénonçait ainsi, en 2006, les incohérences des plans biocarburants, qui «mettent en péril notre sécurité alimentaire » [...], qui « conduisent à minorer la fonction nourricière traditionnelle » (de l'agriculture) au profit d'autres objectifs qui seraient «plus nobles », car plus en phase avec les «attentes de la société ». "C'est, poursuivait l'organisation, dans un tel contexte que prospère le mot d'ordre de la multifonctionnalité de l'agriculture. L'agriculteur trouvera beaucoup plus la valeur ajoutée dont il a impérativement besoin dans les circuits courts qu'en devenant le fournisseur attitré des "raffineries vertes" par des contrats d'approvisionnement rémunérés à un prix marginal peu ou pas $d u$ tout rémunérateur $»$. Ces arguments étaient développés dans le programme de la CR pour les élections de $2007^{18}$. Son président, François Lucas, reconnaissait après les élections que cette position sur les agrocarburants, de même que celle sur les OGM avaient été «deux choses mal comprises » et n'avaient sans doute pas servi le score de l'organisation ${ }^{19}$.

17. «Conquérir de nouveaux marchés » : «Avec la fin programmée des énergies fossiles, de nouveaux horizons s'ouvrent à l'agriculture. Depuis trop d'années, nous n'entendions parler que d'excédents, de surproduction, de gel des terres. Aujourd'hui, il est question de nouveaux débouchés, de nouveaux produits. II faut saisir cette chance, comme il faut relever les défis des biotechnologies, de la chimie verte et des nouveaux matériaux. » (in FNSEA, JA, « Notre métier a un prix... », chapitre 1, $c f$. la note 15 précédemment).

18. «Nourrir les hommes mérite un digne revenu». 19. Entretien de Serge Cordellier avec François Lucas, président de la CR (3 mai 2007, Paris).
En revanche, on avait noté une autre évolution argumentaire, promue par la FNSEA et JA à compter des années 20022003. Elle avait consisté à reprendre l'un des fondements idéologiques de la Coordination rurale : la défense de « prix de marché rémunérateurs », au lieu d'une politique de compensation des revenus par des aides publiques. Luc Guyau, président de l'APCA (Assemblée permanente des Chambres d'agriculture) et précédemment président de la FNSEA (1992-2001), demandait même, dans un article publié le 8 décembre 2005 dans le quotidien Le Monde, que l'avenir commercial international des agricultures du monde se négocie dans le cadre de l'Organisation des Nations Unies pour l'agriculture et l'alimentation (FAO) et non plus dans celui de l'OMC, rejoignant ainsi l'idée d' «exception agriculturelle » de la Coordination rurale.

\section{Identité professionnelle et pratiques syndicales}

\section{Un apanage de la FNSEA et de JA}

La notion de «profession agricole » demeure l'apanage de la FNSEA et de $\mathrm{JA}^{20}$ : elle traduit pour ces deux organisations la volonté d'occuper une position centrale dans l'arbitrage des conflits d'intérêts entre les producteurs des différents secteurs et régions agricoles et de contrôler l'évolution de la définition du titre d'agriculteur et des modèles de conception de l'exploitation agricole. Et la gestion des droits à paiement unique (DPU) institués par la nouvelle PAC en 2003 a été 1'occasion pour eux de relancer le rôle de cogestionnaire qu'ils revendiquent depuis les années 1960, et qui avait été remis en cause dans les années 1990, à la fois par leurs concurrents et par les pouvoirs publics. À travers le suivi et les négociations étroites des dossiers des DPU effectués avec les élus nationaux des Chambres d'agriculture et les services centraux du ministère de l'Agriculture, la

20. Voir à ce sujet Serge Cordellier (2008). 
période 2003-2006 a redonné à la FNSEA et à JA des capacités nouvelles d'influence sur le contrôle des structures agricoles. D'autant que ce travail important d'accompagnement de la mise en place des DPU a réduit les nombreux motifs de mécontentement et de conflit qui étaient apparus sur le terrain entre 2004 et 2006.

\section{Confédération paysanne : \\ un débat récurrent}

En revanche, depuis sa création, en revendiquant le respect du pluralisme syndical, la Confédération paysanne rejette la cogestion qu'elle assimile à une collusion avec l'État et à une confusion de genres et de responsabilités. Même si la question des rapports à l'État (participation aux instances de représentation et de consultation), après avoir longtemps fait l'objet de divergences internes, paraissait plus clairement tranchée en $2007^{21}$, la direction nationale de la Confédération paysanne avait été, après le centrage très médiatisé de ses débats sur l'OMC et les OGM au début des années 2000, moins audible sur les préoccupations professionnelles vécues par les paysans sur le terrain. En outre, la vision du secteur renvoyée aux agriculteurs depuis son niveau national semblait exprimer en creux un «métier réel » à dominante négative : qualité de nourriture dégradée ; environnement menacé ; innovations techniques dangereuses. Vision sans doute mieux comprise à l'extérieur du milieu agricole, en particulier par les médias généralistes, qu'en son sein. Cela aura pu porter certains responsables, notamment parmi les plus sensibles à la dynamique de l'altermondialisme et à la participation aux « mouvements sociaux », à privilégier l'écoute de la société plutôt que l'impact dans le milieu agricole.

21. [Il faut] « continuer d'exercer la représentativité, même ingrate, même contestée par le gouvernement actuel et la FNSEA », in Rapport moral. Projet de contribution au débat stratégique, Assemblée générale de la CP du 19 avril 2006.
La $\mathrm{CP}$ avait aussi peiné à concrétiser ses débats internes, sensibles comme ailleurs entre les régions, les productions, les conceptions du métier, et tiraillés entre marché et protection publique ${ }^{22}$.

Par ailleurs, si la CP est convaincue de proposer un projet global d'alternative ${ }^{23}$, celui-ci est relativement complexe, «contraignant pour les agriculteurs » et « ce n'est pas en cinq minutes qu'on peut l'expliquer » (selon la formule d'une responsable lors de la réunion du Conseil national le 14 février 2007) ${ }^{24}$. En fait, se pose pour cette organisation la question de sa capacité à faire évoluer sa culture professionnelle et politique. Certes, ses résolutions en ont manifesté l'intention, mais le débat a été récurrent au cours de ses vingt années d'existence ${ }^{25}$ et était déjà prévisible dès sa création, quand bien même les sensibilités et clivages internes ont beaucoup changé.

\section{Coordination rurale :}

un discours plus simple

En comparaison, le discours de la Coordination rurale paraît plus simple et plus lisible, même s'il n'est pas pour autant plus opérationnel : protectionniste, antibureaucratique (notamment contre les «apparatchiks de la profession», «ceux qui se servent au lieu de servir »), sécuritaire aux plans alimentaire (garantir

22. Cependant, un dialogue permanent et complexe existe au sein de la $\mathrm{CP}$ entre représentants des zones d'agriculture intensive et responsables issus des zones désertifiées du Sud, souvent «néo-ruraux » (ce dernier terme étant certes très insatisfaisant d'un point de vue scientifique, mais d'emploi commode). 23. Voir son programme pour les élections 2007 : «Paysans, paysannes, reprenons notre avenir en mains».

24. Rapporté par le mensuel de la CP, Campagnes solidaires, $\mathrm{n}^{\circ} 216$, mars 2007.

25. Voir par exemple le rapport moral de l'Assemblée générale de 1992 «Agir, se structurer, pour gagner notre projet » qui plaide en faveur d'un « syndicat d'adhésion » et insiste sur le nécessité de « formation de militants ». 
l'approvisionnement de l'Union européenne) comme non alimentaire (agrocarburants et semences de ferme, paysages préservés) et ruraliste (la survie du monde rural). Si cette organisation dénonce aussi les OGM et la cogestion, elle a cependant mis sur pied, comme la FNSEA dont sont issus beaucoup de ses adhérents d'origine, différentes organisations par produit (Organisation des producteurs de grains, Organisation des producteurs de lait, etc.), qui lui permettent de se battre au plan institutionnel et de prétendre à une crédibilité alternative vis-à-vis du couple FNSEAJA. Elle se montre très attentive aux préoccupations professionnelles. La CR s'est efforcée, par ailleurs, de mettre en place une organisation et une dynamique d'action de terrain (que les organisations concurrentes jugent cependant généralement peu active). Elle essaie, «en se singularisant », de trouver sa place dans une profession dont elle se "sent plutôt exclue car, quand on l'évoque, c'est en général comme propriété du syndicalisme majoritaire $»^{26}$. À l'occasion, elle appelle comme la FNSEA-JA à l' « unité » de ladite profession. Il paraît logique que ces positions aient pu attirer à la Coordination en 2007 des votes qui allaient précédemment à la Confédération.

\section{Les contradictions}

du syndicalisme majoritaire

Comme nous l'évoquions plus haut, on ne peut cependant pas considérer que le syndicalisme majoritaire ait dépassé ses propres contradictions. L'étude de ses déclarations publiques de la période en met plusieurs en évidence : entre libéralisation partielle de la politique des structures, contrôle étroit de la gestion des DPU et demande de protectionnisme ; entre signification sociétale des aides de la PAC et refus de débattre de leur répartition

26. Entretien avec François Lucas déjà cité. entre les agriculteurs ; entre promotion des agrocarburants au nom du développement durable et réticence à l'éco-conditionnalité ; entre ambition de co-animation du groupe Monde rural et recherche de leadership sur « la ruralité ». En revanche, il considère désormais que la diversité des agriculteurs n'est pas un facteur passéiste mais une ressource de développement, et il s'est lancé dans un important travail de communication externe pour rendre l'agriculture «plus attractive».

Enfin, le syndicalisme majoritaire a constamment tenté de répondre à une part importante de son électorat qui exprime, dans son vote comme dans son engagement, une adhésion à finalité d'abord pratique au champ professionnel, et donc une identification qui s'opère parfois par défaut, en combinant une offre de services, une certaine sociabilité locale et une capacité de contre-pouvoir (Le Guen, 2008).

\section{Conclusion}

Quel est le sens des évolutions du vote professionnel agricole de 2007 ? À quelles causes et à quels effets sociologiques renvoient-elles ?

Le regain de participation observé aux élections aux Chambres d'agriculture peut d'abord s'interpréter comme une volonté composite mais forte des agriculteurs de peser sur les orientations des organisations professionnelles agricoles. Ensuite, observés par rapport aux scrutins précédents, les scores des listes syndicales peuvent aussi être analysés comme des comportements d'évaluation de leur offre par les électeurs. Ces derniers s'exprimaient sur un double plan : le degré de pertinence des analyses et des propositions de chaque organisation syndicale dans leur contexte départemental/local ; la capacité de celles-ci à prendre en compte leurs préoccupations professionnelles pour les traduire en orientations positives d'un développement collectif qui soit en phase 
avec les perspectives de marché et les attentes sociétales.

Cette grille d'analyse peut donner sens aux évolutions constatées. La progression des listes FNSEA-JA de 2007, revenant à des proportions voisines de celles 1995, paraît s'expliquer avant tout par des comportements d'électeurs revenus sur leur choix de 2001 en faveur de la CP - mais aussi, dans certains départements, de la $\mathrm{CR}$ - dans un nouveau contexte vécu comme porteur de potentialités et de risques pour le développement économique. Si davantage d'agriculteurs ont voté pour la Coordination rurale en 2007, c'est pour la majorité du fait de la présence, pour la première fois, de listes CR dans leurs départements et, pour d'autres, de la possibilité de passer d'une posture protestataire à une stratégie d'affirmation professionnelle différenciée. Le moindre vote en faveur de la Confédération paysanne paraît lié à son identification à la thématique sociétale, souvent perçue comme passant au second plan les préoccupations économiques et professionnelles.

La question du renouvellement des militants peut aussi pour partie expliquer les flux et reflux des listes entre 2001 et 2007. Outre le vote de nombreux agriculteurs de moins de 35 ans en faveur de JA, la FNSEA bénéficie en effet de l'adhésion de plus des deux tiers des anciens de JA. En revanche, la Confédération paysanne pâtit d'un renouvellement difficile de ses adhérents. Malgré l'intérêt porté aux démarches d'agriculture durable et/ou biologique qu'une partie de ses adhérents incarne et au-delà de sa critique du libéralisme économique (que les autres syndicats portent aussi peu ou prou), les jeunes en phase d'installation ne semblent pas s'identifier en nombre à ses orientations. D'où, dans les années 2000, sa difficulté à assumer la perte, pour raison de départ à la retraite, de nombreux militants syndicalement et politiquement expérimentés qui, formés à la vieille école des solidarités collectives et ayant rompu avec la FNSEA, bénéficiaient d'une image de marque locale fiable, reconnus comme compétents et honnêtes ${ }^{27}$.

Mais le vote de 2007 pose aussi la question de l'évolution du régime d'engagement professionnel des agriculteurs. En reprenant le modèle théorique du sociologue L. Thévenot, nous pouvons émettre l'hypothèse (qu'il reste donc à démontrer) que ce vote résulte d'une réorganisation de ce régime sur trois plans :

- Un engagement de métier : une majorité d'agriculteurs ont exprimé la volonté de rechercher des perspectives nouvelles de développement économique, dans le contexte de marchés agricoles devenus dans l'ensemble plus favorables qu'au début des années 2000.

- Un réengagement collectif : les grandes institutions de la profession agricole essaient de re-légitimer le métier en déplaçant les références des débats publics - centrés dans les années 2000 sur la «malbouffe », les périls affirmés ou contestés de la mondialisation et des innovations génétiques - vers les questions de sécurité alimentaire, de compétitivité internationale des productions européennes et d'émergence de la fonction non alimentaire.

- Un travail identitaire : localement et par leurs organisations, de nombreux agriculteurs travaillent à redéfinir le sens de leur métier, en essayant de substituer à une identité de crise fortement médiatisée - des producteurs dominés économiquement et décalés socialement - une identité de professionnels capables de répondre à de nouvelles attentes sociétales en accomplissant des ruptures à enjeux forts, qu'ils soient techniques, économiques ou environnementaux.

27. Cette question du renouvellement des militants et du vieillissement des cadres a marqué bien plus encore le Modef depuis le tournant des années 1980, même si on a pu constater ces dernières années un net rajeunissement d'une partie de ses cadres nationaux. 
On peut ainsi faire l'hypothèse que les élections de 2007 auraient marqué le passage d'une dynamique professionnelle traversée par le clivage adhésion/protestation à une autre marquée par l'alternative pouvoir/contre-pouvoir. On peut alors comprendre que la conscience qu'exprime une proportion significative des agriculteurs de l'affaiblissement économique et politique de leur profession ait paradoxalement joué en faveur des structures professionnelles dominantes, même si celles-ci ne proposaient pas vraiment de cadre pour répondre à ce déclin. Car, dans un contexte de dérégulation économique, beaucoup d'agriculteurs recherchent, comme on le voit par ailleurs à travers l'évolution des liens coopératifs, des institutions capables de les aider à vivre positivement les changements dont ils sont les acteurs ou qu'ils subissent, qui leur permettent de peser collectivement sur le rapport de force avec les filières agro-industrielles et les pouvoirs publics.

Les cinq derniers scrutins ont progressivement élargi les choix des agriculteurs : ils ne votent plus aujourd'hui seulement «pour» ou « contre » la FNSEA, car ils peuvent être « contre » et choisir une autre façon de voir les choses. La spécificité du scrutin de 2007 est qu'ils recherchent des supports institutionnels facilitant la reconstruction de leur identité professionnelle et la gestion collective de leur hétérogénéité croissante.

\section{RÉFÉRENCES BIBLIOGRAPHIQUES}

Ayats J.-F., Cordellier S., Vincent J., Voisin L.-M. (dir.) (2008). L'univers politique des organisations agricoles. Paris, POUR, $\mathrm{n}^{\circ}$ 196-197.

Becker H.-S. (publié à l'origine en 1960). Notes sur le concept d'engagement. Trad. Camille Debras C., Perdoncin A. (2006). Tracés, Revue de Sciences humaines, $\mathrm{n}^{\circ} 11 / 1$.

Bourrigaud R. (2001). Paysans de LoireAtlantique. 15 itinéraires à travers le siècle. Nantes, Éd. du CHT.

Confédération paysanne (2005). Qu'est-ce que la Confédération paysanne? Paris, Éd. de l'Archipel.

Coordination rurale (2006). Qu'est-ce que la Coordination rurale? Paris, Éd. de l'Archipel.

Cordellier S. (2008). Syndicalisme : du monopole au pluralisme. In «L'univers politique des organisations professionnelles agricole », Paris, POUR, n 196-197.

Cordellier S. (1990). La gauche paysanne moderne et la cogestion. In Coulomb P. et al. (dir.), « Les agriculteurs et la politique », Paris, Presses de Sciences Po.
Coulomb P., Nallet H., (1980). Le syndicalisme agricole et la création du paysan modèle. Paris, Cordes/INRA.

Coulomb P., Delorme H., Hervieu B., Jollivet M., Lacombe P. (dir.) (1990). Les agriculteurs et la politique. Paris, Presses de Sciences Po.

Debatisse M. (1963, 1980). La révolution silencieuse. Paris, Calmann-Lévy.

FNSEA (1984, 1998). 100 ans de syndicalisme agricole. Paris, (nouv. éd.).

Gardella E., Monnet E. (2006). Tracés (éditorial), Revue de Sciences humaines, $n^{\circ} 11 / 1$.

Gaxie D. (dir.) (1985). L'explication $d u$ vote. Paris, Presses de Sciences Po.

Gervais M., Jollivet M., Tavernier Y. (1976). Histoire de la France rurale. Paris, Tome IV, Seuil.

Guyau L. (2004). À quoi sert une chambre d'agriculture? Paris, Éditions de l'Archipel. Hervieu B., Lagrave R.-M. (dir.) (1992). Les syndicats agricoles en Europe. Paris, L'Harmattan.

Lachau J. (1987). Les institutions agricoles. Paris, MA Éditions. 
Lambert B. (1970). Les paysans dans la lutte des classes. Paris, Seuil.

Lavau G. (1981). À quoi sert le Parti communiste français? Paris, Fayard.

Le Guen R. (2008). L'engagement professionnel agricole, entre militantisme et services. Analyse d'une enquête conduite en 2005 auprès de 505 agriculteurs en France. POUR, ${ }^{\circ} 196-197$, p. 114-130.

Le Guen R. (1997). Les élections aux Chambres d'agriculture du 31 janvier 1995, un révélateur du changement d'identité professionnelle des agriculteurs. Paris, Économie rurale, $\mathrm{n}^{\circ} 237$.

Lemétayer J.-M. (2005). Qu'est-ce que la FNSEA ? Paris, Éd. de l'Archipel.

Lemétayer J.-M. (1983). Le pluralisme syndical, une idée neuve ? Repères sur l'histoire du syndicalisme agricole. Paris, AFIP, Matériaux pour la formation, Chemise $\mathrm{n}^{\circ} 4$.

Lemétayer J.-M. (1971). Le syndicalisme agricole hier et aujourd'hui. Paris, Paysans, $\mathrm{n}^{\circ} 85$.

Luneau G. (2004). La forteresse agricole. Une histoire de la FNSEA. Paris, Fayard.

Martin J.-P. (2005). Histoire de la nouvelle gauche paysanne. Des contestations des années 1960 à la Confédération paysanne. Paris, La Découverte.

Martin P. (2001). Comprendre les évolutions électorales. La théorie des réalignements revisitée. Paris, Presses de Sciences Po.

Mayer N. (dir.) (1997). Les modèles explicatifs $d u$ vote. Paris, L'Harmattan.

Mayer N., Perrineau P. (1992). Les comportements politiques. Paris, Armand Colin.
Minot D. (1997). Les élections aux Chambres d'agriculture de 1983. Paris, Économie rurale, $\mathrm{n}^{\circ} 237$.

Muller P. (1984). Le technocrate et le paysan. Paris, Éditions Ouvrières.

Perrineau P., Reynié D. (dir.) (2001). Le dictionnaire du vote. Paris, PUF.

Pesche D., (2000). Le syndicalisme agricole spécialisé en France : entre la spécificité des intérêts et le besoin d'alliances, Paris, L'Harmattan.

Prugnaud L. (1963). Les étapes du syndicalisme agricole. Paris, Éditions de l'Épi.

Purseigle F. (2004). Les sillons de l'engagement. Paris/Marly-le Roi, L'Harmattan/INJEP.

Rémy J. (1982). Le métier d'agriculteur. Façons d'être et façons de produire des agriculteurs sarthois. Paris, INRA-ESR, 2 tomes.

Remy J. (1987). La crise de la professionnalisation en agriculture : les enjeux de la lutte pour le contrôle du titre d'agriculteur. Paris, Sociologie du travail, $\mathrm{n}^{\circ} 4$.

Tavernier Y., Gervais M., Servolin C. (dir.) (1972). L'univers politique des paysans, FNSP/Armand Colin.

Thévenot L. (2006). L'action au pluriel. Sociologie des régimes d'engagement. Paris, La Découverte.

Vercherand J. (1994). Un siècle de syndicalisme agricole. La vie locale et nationale à travers le cas du département de la Loire. Saint-Étienne, Publications de l'Université de Saint-Étienne.

Ysmal C. (1990). Le comportement électoral des Français. Paris, La Découverte, coll. « Repères ». 